\title{
Probiotics: Prevention of Severe Pneumonia and Endotracheal Colonization Trial-PROSPECT: protocol for a feasibility randomized pilot trial
}

Jennie Johnstone ${ }^{1,2,3}$, Maureen Meade ${ }^{4,5}$, John Marshall ${ }^{3,6,7}$, Daren K Heyland ${ }^{8}$, Michael G Surette ${ }^{4}$, Dawn ME Bowdish ${ }^{9}$, Francois Lauzier ${ }^{10}$, Lehana Thebane ${ }^{5,11}$, Deborah J Cook $^{4,5^{*}}$ and For the PROSPECT Investigators and the Canadian Critical Care Trials Group

\begin{abstract}
Background: Probiotics are defined as live microorganisms that may confer health benefits when ingested. Meta-analysis of probiotic trials suggests a $25 \%$ lower ventilator-associated pneumonia (VAP) and $18 \%$ lower infection rates overall when administered to patients in the intensive care unit (ICU). However, prior trials are small, largely single center, and at high risk of bias. Before a large rigorous trial is launched, testing whether probiotics confer benefit, harm, or have no impact, a pilot trial is needed. The aim of the PROSPECT Pilot Trial is to determine the feasibility of performing a larger trial in mechanically ventilated critically ill patients investigating Lactobacillus rhamnosus GG. A priori, we determined that the feasibility of the larger trial would be based on timely recruitment, high protocol adherence, minimal contamination, and an acceptable VAP rate.

Methods/design: Patients $\geq 18$ years old in the ICU who are anticipated to receive mechanical ventilation for $\geq 72$ hours will be included. Patients are excluded if they are at increased risk of probiotic-associated infection, have strict enteral medication contraindications, are pregnant, previously enrolled in a related trial, or are receiving palliative care. Following informed consent, patients are randomized in variable unspecified block sizes in a fixed 1:1 ratio, stratified by ICU, and medical, surgical, or trauma admitting diagnosis. Patients receive $1 \times 10^{10}$ colony forming units of L. rhamnosus GG (Culturelle, Locin Industries Ltd) or an identical placebo suspended in tap water administered twice daily via nasogastric tube in the ICU. Clinical and research staff, patients, and families are blinded.

Discussion: The primary outcomes for this pilot trial are the following: (1) recruitment success, (2) $\geq 90 \%$ protocol adherence, (3) $\leq 5 \%$ contamination, and (4) 10 \% VAP rate. Additional clinical outcomes are VAP, other infections, diarrhea (total, antibiotic associated, and Clostridium difficile), ICU and hospital length of stay, and mortality.

The morbidity, mortality, and cost of VAP underscore the need for cost-effective prophylactic interventions. The PROSPECT Pilot Trial is the initial step toward rigorously evaluating whether probiotics decrease nosocomial infections, have no effect, or actually cause infections in critically ill patients.
\end{abstract}

Trial registration: ClinicalTrials.gov. NCT01782755

Keywords: Critically ill, Intensive care, Probiotics, Infection, Pneumonia

\footnotetext{
*Correspondence: debcook@mcmaster.ca

${ }^{4}$ Department of Medicine, McMaster Health Sciences Center, Room 2C11,

1200 Main Street W, Hamilton, Ontario, Canada

${ }^{5}$ Department of Clinical Epidemiology \& Biostatistics, McMaster University,

Hamilton, Canada

Full list of author information is available at the end of the article
} 


\section{Background}

Ventilator-associated pneumonia (VAP) is the most common nosocomial infection in the intensive care unit (ICU), resulting in a high burden of illness. In a 2005 systematic review, the pooled cumulative incidence of VAP was $23 \%$ (95\% confidence interval (CI) $18.8 \%-$ $26.9 \%$ ) in randomized controlled trials (RCTs) and $10 \%$ (95\%CI 7.0-12.5\%) in observational studies [1]. In addition, VAP is associated with a two-fold attributable risk of dying in the ICU (odds ratio (OR) 2.02, $95 \% \mathrm{CI}$ 1.16-3.56), and the cost attributed to VAP ranges from US $\$ 10,000-\$ 13,000$ per patient [1]. Thus, VAP prevention is an important patient safety goal for critically ill patients [2, 3].

Probiotics, which are live microorganisms thought to have health benefits when ingested, [4] have emerged as a potential strategy to prevent VAP, potentially through enhancing gut barrier function and reducing pathogenic bacterial load [5, 6]. An early systematic review and meta-analysis of RCTs evaluating the efficacy of probiotics on VAP prevention in the ICU conducted in 2010 that included five RCTs showed that probiotics compared with control (placebo or other) reduced the incidence of VAP in mechanically ventilated patients (60/ 316 (19\%) versus 102/373 (27 \%), OR 0.55, $95 \%$ CI $\left.0.31-0.98, I^{2} 39 \%\right)$ [7]. The most rigorous RCT to date compared a combination of oropharyngeal plus gastric Lactobacillus rhamnosus GG to corresponding placebos in 146 patients expected to remain intubated for at least 72 hours (a group at relatively high risk of VAP) [8]. Those patients treated with $L$. rhamnosus GG had lower rates of VAP (17/73 (23\%) versus 33/73 (45\%), relative risk (RR) 0.46, $95 \% \mathrm{CI} 0.26-0.82$ ). This trial suggests that L. rhamnosus GG, specifically, is a promising probiotic to prevent VAP in a selected high-risk ICU population.

Our recent meta-analysis of RCTs in the ICU suggests that probiotics administered to critically ill mechanically ventilated patients are associated with a $25 \%$ lower VAP rate (95\% CI 3-41\%) and $18 \%$ lower infection rates overall (95\% CI 1-31\%) [9]. However, the finding that probiotics may prevent VAP arise from seven small $(n=50-300)$, mostly low quality single-center RCTs yielding imprecise estimates and results with uncertain internal and external validity.

Given the effectiveness of probiotics in the community and hospital setting [10-13], yet uncertain benefits in the ICU, a large rigorous RCT is needed. Before launching a complex costly RCT testing whether probiotics confer benefit, harm, or have no impact on infectious and non-infectious outcomes, a pilot trial is crucial. To this end, we sought to determine whether it is feasible to perform a large RCT in mechanically ventilated critically ill patients to investigate whether orally ingested $L$. rhamnosus GG prevents VAP based on successful and timely pilot trial recruitment, high adherence to protocol, minimal contamination, and an acceptable VAP rate in the Probiotics: Prevention of Severe Pneumonia and Endotracheal Colonization (PROSPECT) Pilot Trial. Ultimately, if the pilot trial is successful, the main trial will aim to determine, among mechanically ventilated critically ill patients, the effect of orally ingested L. rhamnosus GG compared to placebo on VAP and other ICU-acquired infections, diarrhea (including total, antibiotic associated and $\mathrm{C}$. difficile), the duration of mechanical ventilation, ICU and hospital stay, and ICU and hospital mortality (PROSPECT Trial NCT01782755).

\section{Methods/design}

\section{Study design}

We are conducting a parallel group blinded pilot RCT in 14 ICUs in Canada and the United States. Patients are randomized to placebo or probiotic in a fixed allocation ratio of 1:1 using a computer based random number generator produced on the RANDOMIZE.NET website. Randomization is concealed and stratified by ICU and by medical, surgical, or trauma status, in variable unspecified block sizes. Research Coordinators screen all ventilated patients during weekdays. A de-identified log of screened patients is kept, recording each inclusion/exclusion criteria. Reasons for being eligible non-randomized are recorded. Once the Research Coordinator determines that a patient is truly eligible, s/he obtains written informed a priori consent either from the patient or substitute decision maker then notifies the local study pharmacist who performs the randomization.

After randomization, an identical placebo ensures blinding of all possible parties (patient, family, bedside clinicians, laboratory and research personnel, the biostatistician and data analyst).

\section{Eligibility}

Inclusion criteria:

1. Adults $\geq 18$ years old in the ICU;

2. Mechanically ventilated with anticipated ventilation of $\geq 72$ hours at enrolment.

Exclusion criteria:

1. Mechanical ventilation exceeding 72 hours at time of screening;

2. Increased risk of iatrogenic probiotic infection including specific immunocompromised populations (HIV <200 CD4 cells/ $\mu \mathrm{L}$, chronic immunosuppressive medications, prior organ or hematological transplant, neutropenia (absolute neutrophil count $<500$ ); 
3. Increased risk for endovascular infection (history of rheumatic heart disease, congenital heart defect, mechanical valves, endocarditis, endovascular grafts, permanent endovascular devices such as permanent (not short-term) hemodialysis catheters, pacemakers or defibrillators);

4. Mucosal gastrointestinal tract defects (gastroesophageal or intestinal injury, including active bleeding), surgery of the esophagus, stomach, small or large bowel, liver, gallbladder, hepatobiliary tree, spleen, or pancreas within 72 hours, suspected or documented ischemic gut and severe acute pancreatitis, [14]);

5. Strict contraindication or inability to receive enteral medications;

6. Pregnancy;

7. Intent to withdraw advanced life support;

8. Enrolment in this or an ongoing related trial.

\section{Trial interventions}

Patients allocated to the intervention receive $1 \times 10^{10} \mathrm{col}-$ ony forming units (CFU) of L. rhamnosus GG (Culturelle, Locin Industries Ltd) in one capsule suspended in tap water, administered via nasogastric or nasoduodenal tube twice daily in the ICU. L. rhamnosus GG, a variant to $L$. casei rhamnosus is the most widely studied in adults and children [15]. L. rhamnosus GG is approved in Canada for managing and preventing antibiotic-associated diarrhea (Health Canada Natural Product \#80011341). The most rigorous RCT to date investigating probiotics for the prevention of VAP used L. rhamnosus GG as the intervention, and the results of this pilot trial suggest that $L$. rhamnosus GG is a promising option for preventing VAP [8]. Furthermore, recent in vivo studies showed that a dose of L. rhamnosus GG of $\geq 1 \times 10^{9}$ was optimal to reduce bacteremia and mortality from peritonitis and pneumonia [16]. This dose could reduce intestinal epithelial apoptosis and restore colonic epithelial cell proliferation and attenuate the local and systemic inflammatory response, potentially by downregulation of TLR-2/TLR-4 signalling pathway in the colon. [16]. The dose we are using is higher than the dose showing prevention of VAP in the Morrow RCT (total of four billion L. rhamnosus GG) [8] and the same dose as another RCT investigating L. rhamnosus GG in ICU patients as a potential therapy for diarrhea (total of 20 billion L. rhamnosus GG) [17]. Neither trial observed adverse events related to $L$. rhamnosus GG $[8,17]$.

Patients allocated to placebo will receive microcrystalline cellulose, which will also be suspended in tap water, identical in appearance and consistency to the probiotic, and similarly administered. The placebo is prepared by the manufacturer of L. rhamnosus GG, Culturelle, as used in a recent trial [8]. Patients will receive the study product until (1) death or discharge from ICU, or (2) isolation of Lactobacillus spp. cultured from a sterile site or reported as the sole or predominant organism in a culture from a non-sterile site or, (3) 60 days post randomization.

To ensure consistency and document that the probiotic dose matches the label, one capsule from every 10 sheets is being cultured and quantified in the Surette Laboratory at McMaster University.

\section{Outcomes}

The four feasibility outcomes will be:

1. Recruitment of trial patients. Successful recruitment is defined as $\geq 2$ patients per month (total from any study site) on average. This outcome is important because unforeseen enrolment challenges are crucial to identify in a pilot RCT. Unrealistic projected recruitment rates for large studies lead to trial fatigue, threaten non-completion, and generate major cost overruns. Screening logs are being reviewed monthly and eligible non-randomized patients are examined. Barriers to enrolment are discussed and strategies to improve recruitment are being devised. We will calculate the mean (standard deviation) number recruited across all recruiting centers per month.

2. Adherence to protocol. Successful adherence is defined as $\geq 90 \%$ of the prescribed intervention being administered. There are many reasons why ICU patients do not receive drugs administered through the feeding tube. Research Coordinators determine doses actually received and reasons for non-administration using taxonomy. If patients cannot tolerate continuous enteral feeds, they will still usually be able to receive the study product in a small volume of fluid, akin to administering pills or capsule medications. Understandable protocol deviations (e.g., patient died) are being distinguished from protocol violations (e.g., missed doses). We will calculate an overall proportion of doses received divided by total doses prescribed (with $95 \%$ confidence interval).

3. Contamination. Success is defined if $<5 \%$ of patients have any non-study open-label probiotic, in either group, during the ICU stay. The proportion of patients contaminated with any open-label probiotic is being recorded by Research Coordinators daily. We will calculate the total number of patients who ever had open-label probiotics as a proportion (with $95 \%$ confidence intervals) of all randomized patients.

4. VAP. Success is defined if $>10 \%$ patients develop VAP overall. This outcome is important because the widely cited data by quality of care and patient safety initiatives are biased by underreporting, generating 
such low VAP rates that selecting this as a primary trial outcome would make the sample size prohibitively large. A valid contemporaneous rate of VAP will be essential to calculate the sample size for a future large trial. We will calculate the proportion of patients with VAP (with $95 \%$ confidence intervals). Research Coordinators are submitting relevant clinical, radiologic, and microbiologic data to the Methods Center from patients with clinically suspected VAP when there is a new, progressive or persistent radiographic infiltrate with no other obvious cause and the presence of any two of the following symptoms or signs: (1) fever (temperature $>38^{\circ} \mathrm{C}$ ) or hypothermia (temperature $<36^{\circ} \mathrm{C}$ ), (2) relative neutropenia $\left(<3.0 \times 10^{6} / \mathrm{L}\right)$ or leukocytosis $\left(>10 \times 10^{6} / \mathrm{L}\right)$, and (3) purulent sputum [18]. VAP will also be classified several other ways such as clinically suspected and treated VAP, according to the Clinical Pulmonary Infection Score [19] and as adjudicated by independent blinded physicians using previous methods [20-22].

No significance testing between groups is needed to analyze these feasibility objectives. Analyses will be performed at the end of the trial on the cohort of enrolled patients; data will not be divided by allocation group to retain the blinding. No interim analyses or subgroup analyses are planned due to the short duration and sample size of this pilot trial.

\section{Clinical outcomes}

These outcomes are being recorded in the PROSPECT Pilot Trial as well as the main trial.

1. VAP as described above;

2. Other infections defined using an adaptation of the International Sepsis Forum Consensus Conference on Definitions of Infections in the ICU [22];

3. C. difficile-associated diarrhea defined as three or more episodes of unformed stools in $\leq 24$ hours, and C. difficile toxin positive stool or colonoscopic or histopathologic findings demonstrating pseudomembranous colitis [23];

4. Antibiotic-associated diarrhea defined as more than two liquid stools a day for three or more days in quantities in excess of normal for each patient [24];

5. Diarrhea according to the Bristol Stool Chart (1-7);

6. Duration of mechanical ventilation, length of ICU stay, and length of hospital stay;

7. ICU mortality and in-hospital mortality.

\section{Serious adverse events}

The rationale for our approach to serious adverse events (SAEs) accord with our guidelines for academic critical care trials of common interventions [25]. Thus, defined a priori, plausible SAEs have been incorporated as trial outcomes. Any other events that ICU physicians or Site Investigators label as SAEs will be described fully. Any cultures by the ICU team found to be positive for Lactobacillus spp. will have the sample sequenced to determine whether it is consistent with the administered L. rhamnosus GG strain. Lactobacillus spp. identified in a sterile site or cultured as the sole or predominant organism in a non-sterile site will prompt study product discontinuation.

\section{Follow-up}

Patients are being reviewed daily by the Research Coordinator in ICU, where most information is collected. This involves baseline data (e.g., demographics, illness severity, advanced life support), daily data (e.g., study intervention administration and reasons why not administered, nutritional route, relevant medications including antibiotics, agents that may increase gut motility (e.g., prokinetics, laxatives) or decrease gut motility (e.g., opiate infusions), VAP prevention co-interventions, culture results, clinical diagnoses, diarrhea episodes, length of stay, mortality), and Methods Center data (e.g., infection adjudication forms). Any reasons for protocol non-compliance are recorded daily, along with any contamination by non-study probiotic. Co-interventions will likely be comparable between groups, given the blinded design, but they are recorded daily, including other VAP prevention strategies and antibiotics (type, dose, frequency and duration). We do not anticipate any patients lost to follow-up given the hospital time horizon for vital status ascertainment and the ICU stay for key data collection.

\section{Ethics}

The protocol has been approved by each hospital's Research Ethics Board using an a priori consent model. PROSPECT is being conducted in accordance with Good Clinical Practice following the Tri-council Guidelines.

This is an investigator-initiated trial funded from four peer review sources to minimize possible financial conflict of interests. Probiotic and placebo were donated by iHealth, the distributers of Culturelle's L. rhamnosus GG product.

This Pilot Trial does not have a Data Safety and Monitoring Committee (DSMC) due to its size and duration. The larger trial's independent DSMC will create a charter [26] outlining roles, responsibilities, and reporting relationships. For the future large trial, the Trial Biostatistician will provide the DSMC with blinded reports of protocol adherence (e.g., randomization, crossovers, etc.), management indicators (e.g., consent rate, data completion), and clinical outcomes (in interim and final analyses). 


\section{Sample size and recruitment}

Our approach to sample size for a pilot trial focused on feasibility was based on interpreting the lower bound of confidence intervals around estimates [27]-in this case, around the feasibility objective of adherence to protocol, whereby successful adherence is defined as $\geq 90 \%$ of the prescribed intervention being administered. Using patient as the unit of analysis, rather than doses prescribed, the PROSPECT Pilot Trial sample size calculation claims feasibility if at least $80 \%$ of the patients can achieve successful protocol adherence. Recruiting only 150 patients gives an estimate of the proportion of patients with successful adherence with a margin of error between 0.05 and 0.06 in a $95 \%$ CI with a lower bound of 0.84 [Appendix]. However, we increased the sample size into the vanguard Pilot Trial phase to enrol 250 patients in 14 ICUs, for the following reasons: (1) to be sure that at a large number of centers, there is sufficient Research Coordinator exposure to the protocol; (2) to identify any feasibility challenges with data collection methods for the various complex nosocomial infections that could occur at any of the centers with different referral patterns and case mix; and (3) to be sure that we can evaluate our key feasibility objectives in both Canada and the United States [28].

For the future main PROSPECT Trial, the target sample size will be 2650 patients. Based on an estimated $15 \%$ VAP rate, 1325 patients in each group $(n=2650)$ will be enrolled to detect a $25 \%$ relative risk reduction with $80 \%$ power (alpha 0.05 , beta 0.80 ), using a twotailed chi-square test.

\section{Management}

To ensure protocol adherence and data quality, training sessions were held for all research personnel using procedures manuals, standard operating procedures, slide decks, and a study website. The PROSPECT Steering Committee is responsible for the conduct of this PROSPECT Pilot Trial, for upholding or modifying study procedures as needed, addressing challenges with protocol implementation, refining the protocol as needed, and reviewing the data. Steering Committee meetings are held in-person or by conference call quarterly. The Clinical Advances through Research and Information Translation (CLARITY) Research Group at McMaster University is responsible for overall management. We have the requisite breadth and depth of knowledge in study design, implementation, and biostatistics. Our faculty and staff have a wealth of experience in implementation of pilot studies and large international, national, and provincial randomized trials and observational studies. CLARITY has extensive experience and published on calibration, adjudication and efficiencies therein for VAP [21], bleeding [28], lung cancer [29], and thrombosis [30-32]. The
PROSPECT Pilot Trial is conducted under the auspices of the Canadian Critical Care Trials Group [33, 34].

\section{Discussion}

Pilot trials have served the Canadian Critical Care Trials Group well [27, 35], preparing for larger trials. If all four of our PROSPECT Pilot Trial feasibility objectives are attained, we will be well positioned to pursue the main trial. If any of our feasibility objectives are not met, we will rework the protocol in collaboration with the CCCTG and only move forward if the barriers can be properly addressed. The PROSPECT Pilot Trial takes a financially responsible approach to establish research efficiencies and economies of scale before costly grant applications are prepared. If the pilot RCT does not identify a need for any substantive protocol changes, this will be an internal pilot [35]. Accordingly, we will not analyze or report clinical outcomes from the pilot trial; we will include these patient data in the analyses of the main PROSPECT Trial.

Strengths of this proposed trial include rigorous design, inclusion of an inexpensive widely available intervention and multicenter, international enrolment to maximize generalizability. Understanding and reducing potential barriers to enrolment is a critical aim of the PROSPECT Pilot Trial. Rates of consent are influenced by the institutional setting, research infrastructure, and experience of the investigators and research staff [36] as well as physician-specific concerns [37]. It is therefore essential to carefully record and monitor enrolment practices and make improvements where possible. We have successfully conducted other international trials [38-42] and are interested to evaluate co-enrolment across participating centers and countries [43]. When thoughtfully approached, co-enrolment has not been found to impact on trial results $[43,44]$, and thus, ways to maximize safe coenrolment opportunities will be explored in this trial.

It is important to understand whether probiotics are helpful, harmful, or have no effect in vulnerable critically ill patients. The PROSPECT Pilot Trial is foundational to this goal. If the future larger PROSPECT Trial shows that probiotics are effective at preventing VAP, C. difficile, or any infections in the ICU, use may increase. Conversely, if the findings suggest harm or no effect, the reverse is likely. The forthcoming Canadian VAP Prevention Practice Guidelines which currently "suggest" that probiotics are considered in practice (J Muscedere, personal communication, 2015), may, in future updates, either remain the same, change to "recommend" probiotics, or recommend against them, depending on our findings.

\section{Trial status}

Enrolment is underway in the pilot trial. 


\section{Appendix}

\section{PROSPECT Pilot Trial sample size}

The sample size for the PROSPECT Pilot Trial was calculated considering a feasibility objective of adherence to protocol, whereby successful adherence is defined as $\geq 90 \%$ of prescribed intervention being administered. For the sample size of patients, patients are necessarily the unit of analysis rather than doses prescribed. We will consider the trial feasible if at least $80 \%$ of the patients can achieve successful protocol adherence. We based the calculations on the formula for the sample size for a single proportion, based on a confidence interval approach

$$
n \geq \frac{p(1-p)\left(z_{\alpha / 2}^{2}\right)}{M E^{2}}
$$

where $n$ is the required sample size, $p$ is the prior estimate of the proportion of patients with successful protocol adherence, $\mathrm{z}_{\alpha / 2}=1.96$ is the $z$-score, a tabulated value of the standard normal distribution corresponding to a probability value of 0.05 , corresponding to the $95 \%$ confidence interval (CI), and $\mathrm{ME}$ is the margin of error. We created a table providing different values of the sample size $(n)$ based on two prior estimates (PE) of $p, 0.90$ and 0.85 , different margins of error (ME), calculated such that the lower confidence bound (LCB) of the estimated confidence interval estimate will be at least 0.80 . In the PROSPECT Pilot Trial we plan to recruit $n=150$ patients, and based on a prior estimate of $\mathrm{PE}=0.90$, this will give us an estimate of the proportion of patients with successful adherence with margin of error between 0.05 and 0.06 and a $95 \%$ CI with a lower bound of 0.84 .

\section{Abbreviations \\ CCTG: Canadian Critical Care Trials Group; CIHR: Canadian Institute of Health Research; CLARITY: Clinical Advances Through Research and Information Translation; DSMC: Data Safety and Monitoring Committee; ICU: intensive care unit; OR: odds ratio; RCT: randomized controlled trial; RR: relative risk; SAE: serious adverse events; TLR: Toll-like receptor; VAP: ventilator-associated pneumonia.}

\section{Competing interests}

The authors declare that they have no competing interests.

\section{Authors' contributions}

$J J, D K H$, and DJC conceived of the study. JJ, DKH, JM, MGS, DMB, DJC contributed to the background of the study. JJ, MM, JM, FL, DKH, MGS, DMB, $L T$, and DJC participated in the design of the study. MM, JM, FL, DJC acquired the data for the study. DJC, JJ, LT drafted the manuscript. MM, JM, FL, DKH, MGS, DMB revised the manuscript. All read and approved the final manuscript.

\section{Acknowledgements}

The trial was designed by the PROSPECT Steering Committee, the PROTECT Investigators, and the Canadian Critical Care Trials Group. PROTECT was supported by the Canadian Critical Care Trials Group.

We are grateful to Dr. E Petrof and Dr. Paul Wischmeyer who consulted in the planning phases of this trial.

\section{Trial funding}

This trial was funded by the Canadian Institute of Health Research (CIHR), Technology Evaluation for the Elderly, Physicians' Services Incorporated and the Hamilton Academy of Health Sciences Research Organization. iHealth provided the blinded study product. None of these groups played a role in the design, conduct, analysis, interpretation, or write-up of this protocol.

\section{Career funding}

J Johnstone held a CIHR Fellowship award while preparing the protocol. F Lauzier is a recipient of a Research Career Award from the Fonds de la recherche du Québec-Santé. D Cook holds a Chair of the Canadian Institutes of Health Research.

\section{Collaborators}

We appreciate the participation of PROSPECT Collaborators as outlined below:

\section{PROSPECT Pilot Trial clinical collaborators}

- Dr Maureen Meade, Lori Hand, Hamilton Health Sciences, Hamilton General Hospital, Hamilton, Ontario

- Dr John Marshall, Orla Smith, Yoon Lee, Julia Lee, Melissa Wang, St Michael's Hospital, Toronto, Ontario

- Francois Lauzier, Catherine Ouellet, Sarah-Judith Breton, Marie-Claude Tremblay, Stephanie Grenier, Hôpital de l'Enfant-Jésus, Québec City, Québec - Dr Sangeeta Mehta, Brittany Giacomino, Maedean Brown, Sumesh Shah, Marnie Jakab, Mount Sinai Hospital, Toronto, Ontario

- Dr Peter Dodek, Betty Jean Ashley, Victoria Alcuaz, Kelsey Brewer, St. Paul's Hospital, Vancouver, British Columbia

- Dr Lauralyn McIntyre, Irene Watpool, Shelley Acres, Amanda Van Beinum, Ottawa Hospital, General Campus, Ottawa, Ontario

- Dr Joe Pagliarello, Tracy McArdle, Heather Langlois, Rebecca Porteous, Liane LeClaire, Shawna Ready, Brigette Gomes, Ottawa Hospital, Civic Campus, Ottawa, Ontario

- Dr William Henderson, Denise Foster, Susan Logie, Vancouver General Hospital, Vancouver, British Columbia

- Dr Tim Karachi, Ellen McDonald, Hamilton Health Sciences, Juravinski Hospital, Hamilton, Ontario

- Dr Eyal Golan, Margaret Herridge, Andrea Matte, Cheryl Geen-Smith, Brooke Fraser, Sam Kerzner, University Health Network, Western Hospital, Toronto, Ontario

- Dr Gordon Wood, Daniel Okavim, Fiona Auld, Jennifer Good, Vancouver Island Health Authority, Victoria, British Columbia

- Dr Deborah Cook, France Clarke, Lois Saunders, Neala Hoad, St Joseph's Healthcare, Hamilton, Ontario

\section{United States investigators}

- Dr Rodrigo Carten-Ceba, Laurie Meade, Lavonne Liedl, Brenda Anderson, Sueanne Weist, Mayo Clinic, Rochester, Minnesota

- Dr Rob Taylor, Katie Krause, Jackie O'Brien, Kim Fowler, Margaret Cytron, Marianne Tow, St John's Mercy Hospital, St Louis, Missouri

\footnotetext{
Author details

${ }^{1}$ Public Health Ontario, Toronto, Ontario, Canada. ${ }^{2}$ St. Joseph's Health Center, Toronto, Ontario, Canada. ${ }^{3}$ Department of Medicine, University of Toronto, Toronto, Canada. ${ }^{4}$ Department of Medicine, McMaster Health Sciences Center, Room 2C11, 1200 Main Street W, Hamilton, Ontario, Canada. ${ }^{5}$ Department of Clinical Epidemiology \& Biostatistics, McMaster University, Hamilton, Canada. ${ }^{6}$ Department of Surgery, University of Toronto, Toronto, Canada. ${ }^{7}$ Interdepartmental Division of Critical Care, University of Toronto, Toronto, Canada. ${ }^{8}$ Department of Medicine, Queen's University, Kingston, Canada. ${ }^{9}$ Pathology and Molecular Medicine, McMaster University, Hamilton, Canada. ${ }^{10}$ Department of Medicine, Research Center of the $\mathrm{CHU}$ de Québec, Population Health and Optimal Health Practices Research Unit, Québec, Canada. "'Biostatistics Unit, St Joseph's Healthcare-Hamilton, Hamilton, Ontario, Canada.
}

Received: 23 January 2015 Accepted: 13 May 2015

Published online: 24 May 2015 


\section{References}

1. Safdar N, Dezfulian C, Collard H, Saint S. Clinical and economic consequences of ventilator-associated pneumonia: a systematic review. Crit Care Med. 2005:33:2184-93.

2. Muscedere J, Martin C, Heyland D. The direct burden of illness from ventilator associated pneumonia. J Crit Care. 2008;23:5-10.

3. Muscedere J, Dodek P, Keenan S, Fowler R, Cook D, Heyland D, et al. Comprehensive evidence-based clinical practice guidelines for ventilatorassociated pneumonia: prevention. J Crit Care. 2008;23:126-37.

4. Joint FAO/WHO Working Group. Guidelines for the evaluation of probiotics in food: report of a joint FAO/WHO working group on drafting guidelines for the evaluation of probitoics in food, London, Ontario, Canada. 2002. Available at: http://www.fermented-foods.net/wgreport2.pdf. Accessed December 26, 2014.

5. Marshall J. The gastrointestinal flora and its alterations in critical illness. Curr Opin Crit Care. 1999:5:119-25.

6. Brenchley J, Douek D. Microbial translocation across the Gl tract. Annu Rey Immunol. 2012;30:149-73.

7. Siempos I, Ntaidou T, Falagas M. Impact of the administration of probiotics on the incidence of ventilator associated pneumonia: a meta-analysis of randomized controlled trials. Crit Care Med. 2010;38:954-62.

8. Morrow L, Kollef M, Casale T. Probiotic prophylaxis of ventilator-associated pneumonia. Am J Respir Crit Care Med. 2010;182:1058-64.

9. Petrof E, Dhaliwal R, Manzanares W, Johnstone J, Cook D, Heyland D. Probiotics in the critically ill. Crit Care Med. 2012;40(12):3290-302.

10. Hao Q, Lu Z, Dong BR, Huang CQ, Wu T. Probiotics for preventing acute upper respiratory tract infections. Cochrane Database Syst Rev 2011; CD006895.

11. Hempel S, Newberry SJ, Maher AR, Wang Z, Miles J, Shanman R, et al. Probiotics for the prevention and treatment of antibiotic-associated diarrhea: a systematic review and meta-analysis. JAMA. 2012;307:1959-69.

12. Ritchie ML, Romanuk TN. A meta-analysis of probiotic efficacy for gastrointestinal diseases. PLoS One. 2012;7(4), e34938.

13. Johnson S, Maziade PJ, McFarland LV, Trick W, Donskey C, Currie B, et al. Is primary prevention of Clostridium difficile infection possible with specific probiotics? Int J Infect Dis. 2012;16(11):786-92.

14. Ranson J, Rifkind K, Roses D, Fink SD, Eng K, Spencer FC. Prognostic signs and the role of operative management in acute pancreatitis. Surg Gynecol Obstet. 1974;139:69-81.

15. Gorbach S. Probiotics and gastrointestinal health. Am J Gastroenterol. 2000;95:S2-4.

16. Wischmeyer PE, Khailova L. Probiotic administration reduces mortality and improves intestinal epithelial homeostasis in experimental sepsis. Shock. 2012;37:Abstract Supplement.

17. Ferrie S, Daley M. Lactobacillus GG as treatment for diarrhea during enteral feeding in critical illness: randomized controlled trial. J Parenter Enteral Nutr. 2011;35:43-9.

18. Grossman R, Fein A. Evidence-based assessment of diagnostic tests for ventilator-associated pneumonia: executive summary. Chest. 2000;117:1775-81.

19. Pugin J, Auckenthaler P, Mill N, Janssens JP, Lew D, Suter PM. Diagnosis of ventilator-associated pneumonia by bacteriologic analysis of bronchoscopic and nonbronchoscopic "blind" bronchoalveolar lavage fluid. Am Rev Respir Dis. 1991;143:1121-9.

20. Heyland D, Muscedere J, Wischmeyer P, Cook DJ, Jones G, Elke G, et al. A randomized trial of high dose glutamine and anti-oxidants in critically ill patient with multi-organ failure. N Engl J Med. 2013;368:1489-97.

21. Cook D, Walter S, Cook R, Griffith L, Guyatt G, Leasa D, et al. Incidence of and risk factors for ventilator-associated pneumonia in critically ill patients. Ann Intern Med. 1998;129:433-44.

22. Calandra T, Cohen J. For the International Sepsis Forum Definition of Infection in the ICU Consensus Conference. Crit Care Med. 2005;7:1538-48.

23. Cohen S, Gerding D, Johnson S, Kelly C, Loo V, McDonald L, et al. Clinical practice guidelines for Clostridium difficile infection in adults: 2010 update by the Society for Healthcare Epidemiology of America (SHEA) and the Infectious Diseases Society of America (IDSA). Infect Control Hosp Epidemiol. 2010;31:431-55.

24. Hickson M, D'Souza AL, Muthu N, Rogers T, Want S, Rajkumar C, et al. Use of probiotic Lactobacillus preparation to prevent diarrhoea associated with antibiotics: randomised double blind placebo controlled trial. BMJ. 2007;335(7610):80
25. Cook D, Lauzier F, Rocha MG, Sayles MJ, Finfer S. Serious adverse events in academic critical care research. CMAJ. 2008;178:1181-4.

26. DAMOCLES Study Group. A proposed charter for clinical trial data monitoring committees: helping them to do their job well. Lancet. 2005;365:711-22.

27. Thabane L, Ma J, Chu R, Cheng J, Ismaila A, Rios LP, et al. A tutorial on pilot studies: the what, why and how. BMC Med Res Methodol. 2010;10:1. http://www.biomedcentral.com/1471-2288/10/1.

28. Arnold DM, Lauzier M, Rabbat C, Zytaruk N, Barlow-Cash B, Clarke F, et al. Adjudication of bleeding outcomes in an international thromboprophylaxis trial in critical illness. Thromb Res. 2013;131:204-9.

29. Walter SD, Cook DJ, Guyatt G, King DB, Troyan S. Outcome assessment for clinical trials: how many adjudicators do we need? Control Clin Trials. 1997; 18:27-42.

30. Heels-Ansdell D, Saunders L, Zytaruk N, Southon J, Barlow Cash B, Lutz K, et al. Methods center personnel costs for adjudication of 5 morbidity outcomes in a thromboprophylaxis trial. Am Rev Resp Crit Care Med. 2011;183:A1678

31. Zytaruk N, Meade M, Mehta S, Hall R, Southon J, Heels-Ansdell D, et al. Adjudication calibration for pulmonary embolism in a thromboprophylaxis trial. Am J Resp Crit Care Med. 2009:179:A1582.

32. Dodek FLLMIP. Calibration and adjudication of thrombotic events in a thromboprophylaxis trial Canadian Critical Care Forum abstract book. 2009

33. Cook DJ, Brower R, Cooper J, Brochard L, Vincent J-L. Multicentre clinical research in critical care. Crit Care Med. 2002;30:1636-43.

34. Marshall JC, Cook DJ, Canadian Critical Care Trials Group. Investigator-led clinical research consortia: the Canadian Critical Care Trials Group. Crit Care Med. 2009;36(7):S165-72.

35. Arnold DA, Burns KE, Adhikari N, Kho ME, Meade MO, Cook DJ. The design and interpretation of pilot trials in critical care medicine. Crit Care Med. 2009;36(7):S69-74

36. Smith OM, McDonald E, Zytaruk N, Doster D, Matte A, Clarke F, et al. Rates and determinants of informed consent: a case study of an international thromboprophylaxis trial. J Crit Care. 2013;28:28-39.

37. Cook D, Arabi Y, Ferguson N, Heels-Ansdell D, Freitag A, McDonald E, et al. Physicians declining patient enrollment in a critical care trial: A Case Study in thromboprophylaxis. Intensive Care Med. 2013;39:2115-25.

38. Lacroix J, Hébert PC, Fergusson D, Tinmouth A, Cook D, Marshall J, et al, for the ABLE investigators*and the Canadian Critical Care Trials Group. The Age of Blood Trial in Critically ill Adults. N Engl J Med 2015; DOI:10.1056/ NEJMoa1500704

39. Heyland D, Muscedere J, Wischmeyer P, Cook D, Jones G, Albert M, et al. A randomized trial of high dose glutamine and anti-oxidants in critically ill patient with multi-organ failure. N Engl J Med. 2013;368:1489-97.

40. Ferguson ND, Cook DJ, Guyatt GH, Mehta G, Hand L, Austin P, et al. High-frequency oscillation in early acute respiratory distress syndrome. N Engl J Med. 2013;368(9):798-805.

41. Mehta S, Burry LD, Cook DJ, for the SLEAP Investigators and the Canadian Critical Care Trials Group, et al. Daily sedation interruption in mechanically ventilated critically ill patients cared for with a sedation protocol: A randomized controlled trial. JAMA. 2012;308(19):1985-92.

42. Cook DJ, Meade M, Guyatt G, for the PROTECT (Prophylaxis for ThromboEmbolism in Critical Care Trial) Investigators for the Canadian Critical Care Trials Group, the Australian and New Zealand Intensive Care Society Clinical Trials Group, et al. A Randomized Trial of Dalteparin versus Unfractionated Heparin in Critically III Patients. N Engl J Med. 2011;364(14):1305-14.

43. Cook D, McDonald E, Smith O, for the PROTECT Investigators and the Canadian Critical Care Trials Group, et al. Co-enrollment of critically ill patients into multiple studies: patterns, predictors and consequences. Crit Care. 2013:17:R1

44. Cook DJ, Hand L, Austin P, for the OSCILLATE Investigators and the Canadian Critical Care Trials Group, et al. Coenrolment in a randomized trial of high frequency oscillation in ARDS: Prevalence, patterns, predictors and outcomes. Crit Care Med. 2014;43(2):328-38. 